\title{
Responsabilidad Social Universitaria en Educación Mediada por Tecnologías en Contexto de Pandemia COVID-19
}

\author{
University Social Responsibility in Technology Mediated Education in the Context of \\ the COVID-19 Pandemic
}

Gretser José Orellana-Méndez

\section{(2) \\ EDICIÓN: \#EIVTAC}

Recibido: 25/junio/2021

Aceptado: $18 /$ septiembre/2021

Publicado: 24/septiembre/2021

我 País

${ }^{1}$ Venezuela

\section{IIIII Institución}

${ }^{1}$ Universidad Yacambú

\section{Correo Eletrónico}

'19retserorellana@gmail.com

\section{(iD) ORCID}

'https://orcid.org/0000-0001-6090-5192

\section{Citar así: CFAPA / IEEE}

Orellana-Méndez, G. (2021) Responsabilidad Social Universitaria en Educación Mediada por Tecnologías en Contexto de Pandemia COVID-19. Revista Tecnológica-Educativa Docentes 2.0, 11(2), 28-39. https://doi.org/10.37843/rted.v11i2.247

G. Orellana-Méndez, "Responsabilidad Social Universitaria en Educación Mediada por Tecnologías en Contexto de Pandemia COVID-19", RTED, vol. 11, n. ${ }^{\circ} 2$, pp. 28 39, sep. 2021.

\section{Resumen}

La universidad es una comunidad de intereses donde los procesos educativos tienen como médula acciones emergentes dentro de su dinámica institucional. Por ello, el objetivo de la investigación fue interpretar experiencias de docentes de la Universidad Yacambú sobre la responsabilidad social universitaria (RSU) en educación mediada por tecnologías asociada a la pandemia COVID-19. En este sentido, esta investigación se realizó bajo un enfoque cualitativo de paradigma interpretativo. El plano epistemológico fue asumido desde el construccionismo social para así comprender los significados emergidos del intercambio dialógico entre el investigador e informantes clave, todo ello con apoyo del método fenomenológicohermenéutico. La muestra partido del arqueo de opiniones de 4 profesores adscritos a Facultad de Humanidades de la Universidad Yacambú con funciones de docencia, investigación, extensión, gestión. Como hallazgo de esta investigación, se obtuvieron que las universidades son instituciones de gran relevancia para el desarrollo social de una nación, pues responde a necesidades de poblaciones, atendiendo a problemáticas emergentes producto de una actividad investigativa, logrando así reivindicar su papel de responsabilidad social. Igualmente, se concluyó con la determinación de lo importante del aspecto tecnológico para una consolidación del docente y estudiante en su contexto actual.

Palabras clave: Pandemia COVID-19, responsabilidad social universitaria, educación mediada por tecnologías.

\section{Abstract}

The university is a community of interests where educational processes are their core emerging actions within their institutional dynamics. Therefore, the research objective was to interpret the experiences of professors from the Yacambú University on university social responsibility (USR) in education mediated by technologies associated with the COVID-19 pandemic. In this sense, this carried research was out under a qualitative approach of an interpretive paradigm. The epistemological plane was assumed from social constructionism to understand the meanings that emerged from the dialogical exchange between the researcher and key informants, all with the support of the phenomenological-hermeneutical method. The sample is based on the archiving of opinions of 4 professors assigned to the Faculty of Humanities of the Yacambú University with functions of teaching, research, extension, management. As a finding of this research, it was obtained that universities are institutions of great relevance for the social development of a nation since they respond to the needs of populations, attending to emerging problems resulting from an investigative activity, thus managing to vindicate their role of social responsibility. Likewise, it was concluded to determine the importance of the technological aspect for a consolidation of the teacher and student in their current context.

Keywords: COVID-19 pandemic, university social responsibility, technology-mediated education. 


\section{Introducción}

La universidad es una comunidad de intereses donde los procesos educativos tienen como médula acciones emergentes dentro de su dinámica institucional. La tecnificación del proceso educativo lleva más de una década, pero ha tenido un vertiginoso crecimiento en los dos últimos años debido a la pandemia por CODIV-19, donde el distanciamiento social ha sido una medida tomada por diversos países a fin de mitigar su tasa de contagio entre personas.

De acuerdo con Núñez et al. "La redimensión del espacio-tiempo formativo, involucra que las herramientas tecnológicas no presenciales también permiten establecer vínculos relacionales formativos" $(2019$, p.7), este planteamiento apoya los procesos a través de una educación mediada por tecnologías, donde su vertiente no debe esgrimirse solamente al proceso de docencia, sino trascender a todas las funciones sustantivas universitarias. Por ello, la Responsabilidad Social Universitaria (RSU) es un eje dinamizador que permite desarrollar el planteamiento previamente descrito.

En este sentido, una forma de vincular a la universidad con su contexto es mediante procesos de RSU, un concepto derivado a partir de Responsabilidad Social Empresarial (RSE). Por ello, Vallaeys destaca “....es una nueva política de gestión universitaria que se va desarrollando en Latinoamérica para responder a los impactos organizacionales y académicos de la universidad.... la RSU no es cómoda, puesto que fuerza a la autocrítica institucional" (2014, p.1). Este concepto invita a desarrollar diversas políticas para ser visibles tanto nacional como internacionalmente, para así ser instituciones competitivas en un mercado tan amplio como el del capital intelectual, el cual incluye a su vez su forma de gestionar el conocimiento a partir sus experiencias prácticas.

Como apoyo a RSU, la Constitución de la República Bolivariana de Venezuela establece en su artículo 132 "toda persona tiene el deber de cumplir sus responsabilidades sociales y participar solidariamente en la vida política, civil y comunitaria del país, promoviendo y defendiendo los derechos humanos como fundamento de la convivencia democrática y de la paz social" (1999, p. 65), este principio rector constitucional invita ser ciudadanos con el deber de fortalecer su responsabilidad social, a través de un ente generador de conocimientos como lo representan universidades, quienes propician el fortalecimiento de valores éticos para una formación activa en procesos de intercambio de experiencias en la construcción de alianzas comunidad-universidad para beneficios mutuos en pro del desarrollo del Estado.

En el devenir de su gestión, esta casa de estudios se encarga de la formación de profesionales en diversas aéreas del conocimiento, tales como humanidades, ingenierías, administrativas a nivel de pregrado, maestrías, doctorado en gerencia, donde desde hace ya más de quince años se ha sumado con gran interés a la modalidad de estudios a distancia a través del apoyo en Tecnologías de la Información y la Comunicación (TIC), con el firme propósito de satisfacer necesidades sociales, al brindarles a sus educandos, conocimiento, innovación, ventajas competitivas, preparándolos para solucionar problemas de su entorno, destacando con mayor importancia por la situación de pandemia de COVID-19.

Ahora bien, al retomar el constructo sobre Responsabilidad Social Universitaria, Muñoz expresar "La... (RSU) necesita pensarse en base a dos hechos contemporáneos: la situación social y ecológica global y la masificación de la Educación Superior" (2012, p.5); estas dos vertientes se manifiestan en el desarrollo tanto de política como de gestión universitaria para atender demandas del mundo globalizado, incorporándose en estándares de calidad, manejo de recursos institucionales en escenarios endógenos y exógenos. Por ello, el objetivo de la investigación fue interpretar experiencias de docentes de la Universidad Yacambú sobre la responsabilidad social 
universitaria (RSU) en educación mediada por tecnologías asociada a la pandemia COVID-19.

\section{Metodología}

Desde que el ser humano desarrolló capacidad de raciocinio, ha estado en una constante búsqueda de respuestas a problemas inmersos en su realidad o entorno. En el presente estudio, se hizo un abordaje ontoepistemológico y metodológico fundamentado en intersubjetividad a fin de generar una postura teórica sobre el fenómeno de estudio, para así comprender una construcción emergente de responsabilidad social universitaria desde educación mediada por tecnología producto de la pandemia COVID-19, a través de funciones de extensión, investigación, docencia y gestión desde percepciones de informantes clave.

Al relacionar RSU con educación mediada por tecnologías, se está indagando desde su génesis en cómo cada universidad lleva a cabo esta práctica desde sus propios participantes hasta la forma de administrar los saberes de su capital humano, el cual estará en sintonía con sus políticas declaradas para responder a necesidades determinadas en sus planes de acción de intervención sociocomunitaria.

En esta línea temática, el trabajo se asume desde el paradigma interpretativo con enfoque cualitativo. Asimismo, se concibe desde una visión fenomenológica, Pérez (1994), señala como una característica importante de este paradigma que la teoría constituye una reflexión en desde su praxis, conformando una realidad de hechos observables y externos, por significados e interpretaciones elaboradas del propio sujeto, a través de interacciones con los demás dentro de un contexto determinado. Por ello se enfatiza en la comprensión de realidades desde creencias, valores, reflexiones de informantes clave.

De acuerdo con Orellana "Para conducir esta investigación basada en un método fenomenológico-hermenéutico, fueron necesarios una serie de pasos, desde la entrada hasta la salida del camino de trabajo, que se tienen de forma previa y otros que fueron emergiendo en la medida que se desarrolle el proceso" (2015, p.94), este hecho se aplica de forma textual al fenómeno en estudio, debido a lo recursivo del método empleado.

Por su parte, Martínez (2006) divide en el abordaje del método en las etapas previa, descriptiva, estructural y de discusión de los resultados. Todas estas fases se llevaron a cabo para así interpretar los hallazgos obtenidos luego del intercambio dialógico con cada una de las informantes clave de esta investigación, quienes expresaron su punto de vista de cómo se ha desarrollado responsabilidad social universitaria desde un enfoque educativo mediado por tecnologías, producto del COVID-19 en la Universidad Yacambú.

De estas ideas se puede realizar un análisis profundo; pero sencillamente lo importante es asumir de forma categórica el rol que tiene el investigador desde su papel protagónico conferido por sus informantes, quienes realmente son coinvestigadoras, por cuanto ellas hicieron reflexiones sobre sus vivencias, experiencias y participación como constructoras de una visión acerca del fenómeno de estudio, partiendo de una relación dialógica de partes interactuantes. En Figura 1 se muestra un gráfico con diagramación de coherencia metodológica.

Ahora bien, para saber quiénes son los protagonistas de esta investigación, se define a los informantes claves según Martínez como "personas con conocimientos especiales y buena capacidad de información" (1991, p. 56). Por ello, el investigador realizó un arqueo de opiniones entre los profesores adscritos a Facultad de Humanidades de la Universidad Yacambú con funciones de docencia, investigación, extensión, gestión. Asimismo, con disposición de participar aportando sus visiones y experiencias. Las características de cada una de ellas se describen en Tabla 1: 


\section{Figura 1}

Matriz ontoepistémica y metodológica

Plano Ontológico: Interpretativo dialógico

Plano Epistemológico: Construccionismo Social

Plano Metodológico: Fenomenológico Hermenéutico

Nota. Coherencia metodológica de la investigación, elaboración propia (2021).

Tabla 1

Identificación de las informantes clave

\begin{tabular}{|c|c|c|c|}
\hline $\begin{array}{l}\text { Informante } \\
\text { Clave }\end{array}$ & Identificación & Rol & $\begin{array}{l}\text { Tiempo } \\
\text { servicio }\end{array}$ \\
\hline IC1 & NA & Docente de Facultad de Humanidades & Tres años \\
\hline IC2 & LA & $\begin{array}{l}\text { Docente de Facultad de Humanidades- } \\
\text { Coordinadora de Línea de Investigación }\end{array}$ & Cinco años \\
\hline IC3 & YS & $\begin{array}{l}\text { Docente y Enlace de Servicio Comunitario en } \\
\text { Facultad de Humanidades }\end{array}$ & Tres años \\
\hline
\end{tabular}

Nota. Atributos de informantes clave, elaboración propia (2021).

Las técnicas e instrumentos de recolección de información constituyen una parte importante en metodología de una investigación. En este trabajo se empleó entrevista en profundidad, Martínez destaca como una técnica que adopta una forma de diálogo coloquial de gran relevancia, “...a medida que el encuentro avanza, la estructura de la personalidad del interlocutor va tomando fuerza en nuestra mente; adquirimos las primeras impresiones con la observación..." (2006, p. 93); este hecho fue evidenciado por el investigador al momento de realizar esta actividad como medio para obtener información de los informantes clave, quienes a medida del transcurso del tiempo, fueron entrando en confianza, fluyendo más rápidamente en su discurso. Como apoyo al proceso de entrevista se utilizó un guion de preguntas generadoras y se grabó esa información, previa autorización de cada informante.

Para el proceso de interpretación de categorías emergentes se trabajó con la triangulación, que es una técnica empleada para lograr los propósitos planteados. Sobre esta se tiene:
La triangulación se refiere al uso de varios métodos (tanto cuantitativos como cualitativos), de fuentes de datos, de teorías, de investigadores o de ambientes en el estudio de un fenómeno... Este término metafórico representa el objetivo del investigador en la búsqueda de patrones de convergencia para poder desarrollar o corroborar una interpretación global del fenómeno humano objeto de la investigación... (Okuda et.al, 2005, p.119)

Como se evidencia en definición previa, en esta investigación se buscó convergencia de opiniones sobre responsabilidad social universitaria desde una educación mediada por tecnologías en contexto de pandemia por COVID-19 en la Universidad Yacambú. La acción descrita la realizó el investigador a partir de los testimonios de tres informantes clave, donde se generaron categorías y hallazgos relevantes desarrollados en los resultados.

\section{Resultados}

Como resultado de los propósitos de investigación establecidos, se presentan los hallazgos producto de una sistematización y categorización del discurso de cada uno de los actores universitarios, obtenido luego de 
transcripción de entrevista en profundidad realizada a cada uno. Las informaciones fueron plasmadas en una matriz contentiva de identificación del informante clave, línea, verbatum o discurso del entrevistado, categoría, subcategoría y código. Al mismo tiempo, se realizó el proceso de cromatización de los parlamentos donde el investigador identificó las categorías como subcategoría, asimismo fueron codificadas en una matriz.

Posterior a esta acción, se procedió a realizar comprensión de los significados emergentes por cada categoría según percepción de los diferentes informantes clave, a través de una confrontación de unidades de significado asociadas a la categoría respectiva. Dicha comprensión se generó a partir abstracciones de los juicios realizados por el investigador para adentrase en el pensamiento de los sujetos. Esta narrativa permitió encontrar un sentido de la información, para luego realizar el proceso de interpretación, en donde se realiza triangulación a partir de tres fuentes: (a) Unidad de significado de los actores universitarios, (b) Posición de los referentes teóricos sobre la temática, (c) El análisis aportado por el investigador. Del proceso hermenéutico surgió como primera categoría denominada "Universidad como Ser Viviente" con dos subcategorías (ver Tabla 2), donde se destaca el rol trascendental de las universidades, quiénes son entes dinámicos con naturaleza evolutiva y tendencia a estar en sintonía con necesidades presentes en su entorno, así como los requerimientos surgidos de su vinculación con las funciones universitarias.

Tabla 2

Primera categoría emergente

\begin{tabular}{|c|c|c|}
\hline Categoría & Subcategorías & Hallazgos \\
\hline \multirow[t]{2}{*}{$\begin{array}{l}\text { Universidad como } \\
\text { Ser Viviente (USV) }\end{array}$} & $\begin{array}{l}\text { Valores de institución universitaria } \\
\text { (VIU) }\end{array}$ & $\begin{array}{l}\text { - Respeto a ideas } \\
\text { - Humildad } \\
\text { - Trabajo social } \\
\text { - Responsabilidad social } \\
\text { - Cuidado ambiental } \\
\text { - Desarrollo humanista }\end{array}$ \\
\hline & $\begin{array}{l}\text { Universidad de puertas abiertas } \\
\text { (UPA) }\end{array}$ & $\begin{array}{l}\text { - Evolución y aprendizaje } \\
\text { - Infraestructura al servicio de sociedades }\end{array}$ \\
\hline
\end{tabular}

Nota. Representación de proceso de categorización emergente, elaboración propia (2021).

Al ver a la universidad como un ser viviente, se debe analizar en su constitución funciones que delimitan una acción educativa en el país, docencia, investigación y extensión son esas líneas guías en planificación de procesos para una comunidad universitaria, todas articuladas en el intercambio de aportes a través de la gestión. Esta última es un proceso direccionador de políticas y planes operativos de cada institución universitaria, por ello es pertinente vincular este planteamiento con lo mencionado por Baca quien explica cómo “... La RSU responde al pilar social de la sostenibilidad universitaria en un largo plazo..." (2015, p.208). En este sentido, un aporte refiere cómo RSU es fundamental en una dinámica de instituciones educativas de nivel superior, pues se traduce en el compromiso con el entorno, más una formación de calidad en los profesionales cursantes de pregrado y postgrado.

Como segunda categoría emergida, el currículo como medio para RSU (ver Tabla 3 ), donde se pone en manifiesto el papel del currículo para consolidar una vinculación de los planes de estudio de cada carrera con el compromiso social implícito en instituciones de educación superior del país, al tiempo se evidencia una multimodalidad de formas de administración educativa, donde, por ejemplo está una modalidad mediada por TIC. 
Tabla 3

Segunda categoría emergente

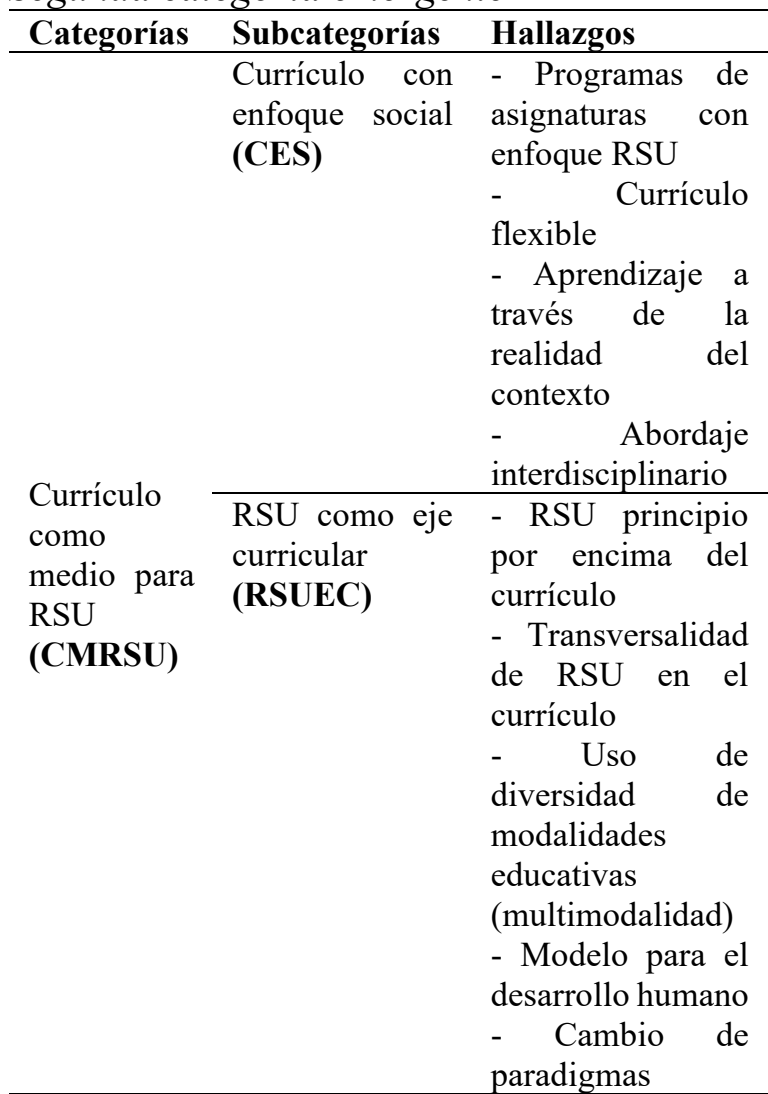

Nota. Representación de proceso de categorización emergente, elaboración propia (2021).

Al relacionar los aspectos inmersos en esta segunda categoría con lo planteado por Vallaeys (2014), quien señala la existencia de cuatro impactos que definen cuatro procesos de gestión socialmente responsable de universidades, divisibles en dos ejes, uno organizacional y otro académico. Estas particularidades son base fundamental para tener en evidencia el rol de responsabilidad social en universidad evitando así su confusión con procesos empresariales. En este sentido, el eje organizacional relaciona los impactos internos-externos de cada universidad definidos por los aspectos laborales-ambientales. A su vez el eje académico es el puente entre los impactos educativos con los cognitivos.

Producto de los planteamientos anteriores, Vallaeys, señala "estos 4 impactos definen cuatro ejes de gestión socialmente responsable de la Universidad, a saber: (a) Campus responsable; (b) Formación ciudadana y profesional responsable; (c) Gestión social del conocimiento; (d) Comunidades de aprendizaje mutuo para el desarrollo" (2007, p.8-9). De esto, el mayor énfasis se da en el proceso de formación ciudadana como profesional responsable, en donde Vallaeys incluye lo curricular para formar a un profesional con sentido humanista con el apoyo de orientación curricular general como parte de los cursos de cada carrera. Asimismo, es necesario se tenga una relación estrecha con los problemas reales de desarrollo (económicos, sociales, ecológicos, entre otros) en contacto directo con actores externos a la comunidad universitaria.

Como se logra evidenciar en este discurso, el papel del currículo es fundamental para consolidar una responsabilidad social universitaria como parte dinámica de la formación profesional del estudiante. Asimismo, al comprender el discurso de informantes clave se pudo extraer una serie de ideas acerca del fenómeno en estudio. Algunos planteamientos tienen relación con los programas de asignaturas orientados hacia un enfoque de RSU, el currículo amerita ser flexible para desarrollarse en un ámbito dinámico, logrando obtener un aprendizaje a través de realidades del contexto, abordado con una perspectiva interdisciplinaria a la luz de diferentes estrategias acordes con la modalidad educativa asumida, sea presencial o mediada por tecnologías.

La categoría "el estudiante dentro del contexto comunitario" denota su importancia como partícipe en el proceso de responsabilidad social, debido a que tiene un papel protagónico en la construcción de su conocimiento a partir de experiencias útiles como persona y profesional en formación. En Tabla 4 se presentan subcategorías emergidas de voces de informantes clave en torno a esta categoría orientadora. 


\section{Tabla 4}

Tercera categoría emergente

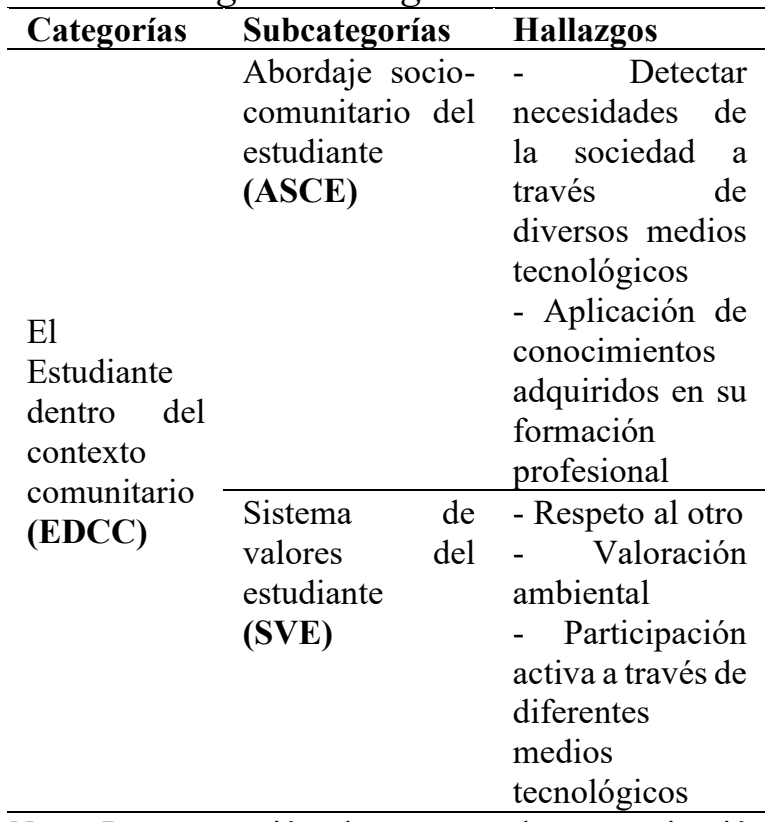

Nota. Representación de proceso de categorización emergente, elaboración propia (2021).

Dentro de la teoría de RSU los estudiantes son extraídos de dimensiones tradicionales de universidad (docencia, investigación, extensión), porque éstos se encuentran formando parte de diversos procesos en instituciones de educación superior; ya sea cursando estudios de pre o postgrado (representa docencia); estudian realidades (investigan); también realizan actividades con comunidades, haciendo extensión en representación de su universidad. Por este motivo queda claro el importante rol del estudiante como agente socializador del conocimiento.

"Experiencias Comunitarias" es una categoría que reúne todos aquellos testimonios vinculados al acercamiento de los integrantes de la comunidad universitaria con su sociedad, por ello, emergieron tres subcategorías como son: formación $\mathrm{y}$ orientación comunitaria, aprendizaje colaborativo en valoración de experiencia, servicio comunitario proceso de acción continua (ver Tabla 5).
Tabla 5

Cuarta categoría emergente

\begin{tabular}{|c|c|c|}
\hline Categorías & Subcategorías & Hallazgos \\
\hline \multirow{3}{*}{$\begin{array}{l}\text { Experiencias } \\
\text { comunitarias } \\
\text { (EC) }\end{array}$} & $\begin{array}{l}\text { Formación } \\
\text { orientación } \\
\text { comunitaria } \\
\text { (FOC) }\end{array}$ & $\begin{array}{l}\text { - Atención de } \\
\text { necesidades } \\
\text { comunitarias } \\
\text { desde } \\
\text { experiencias } \\
\text { del estudiante } \\
\text { - Asistencia a } \\
\text { comunidades }\end{array}$ \\
\hline & $\begin{array}{ll}\text { Aprendizaje } & \\
\text { colaborativo en } & \text { en } \\
\text { valoración } & \text { de } \\
\text { experiencias } & \\
\text { (ACVE) } & \end{array}$ & $\begin{array}{l}\text { - Valoración } \\
\text { del aprendizaje } \\
\text { obtenido en } \\
\text { vivencias } \\
\text { comunitarias } \\
\text { - Inversión de } \\
\text { tiempo } \\
\text { recursos } \\
\text { - Diálogo de } \\
\text { saberes en } \\
\text { comunidades }\end{array}$ \\
\hline & $\begin{array}{l}\text { Servicio } \\
\text { Comunitario } \\
\text { proceso de } \\
\text { acción continua } \\
\text { (SCPAC) }\end{array}$ & $\begin{array}{l}\text { - Diseño de } \\
\text { proyectos de } \\
\text { acción } \\
\text { - Continuidad } \\
\text { del abordaje } \\
\text { comunitario } \\
\text { - Arista de RSU }\end{array}$ \\
\hline
\end{tabular}

Nota. Representación de proceso de categorización emergente, elaboración propia (2021).

Formación y orientación continua es una subcategoría que representa parte de responsabilidad social universitaria, desde su atención en comunidades a través de diferentes programas educativos $\mathrm{o} d \mathrm{de}$ formación para brindar herramientas a las personas a través de orientaciones brindadas por los estudiantes en relación con necesidades detectadas en su área de competencia.

El servicio comunitario no es el único lazo entre universidad y su entorno, sino que es una parte de su dinámica. También, en ocasiones se tiene como falsa creencia como esta acción de ley es responsabilidad social, en parte lo es, pero no representa una única vía de acción, por eso se invita reflexionar sobre los procesos asociados al servicio comunitario de cada institución de educación superior estén definidos en reglamentos y de esta manera haya un direccionamiento hacia dónde van $a$ ir orientadas acciones a 
desarrollar el estudiantado, para que puedan abordar los problemas sociales desde asignaturas, trabajos de grado $\mathrm{u}$ otra actividad curricular existente a lo largo de su formación profesional.

Para reforzar algunas ideas previas, el autor Hernández vincula su postura sobre atención comunitaria y valoración de experiencias cuando señala "la gestión del conocimiento es un concepto aplicado en las organizaciones, y hace referencia a la transmisión del conocimiento y de la experiencia existente entre sus miembros" (2014, p.9). En este sentido, los conocimientos son un recurso a disposición de los miembros de una organización, donde la construcción colectiva a partir de experiencias en cada actor clave del proceso, lo que se deja en evidencia al momento de valoración de experiencias comunitarias, pues se generan conocimientos gestionables que se transmiten a un colectivo de interés.

Como quinta categoría sustentada en los hallazgos, se tiene, praxis gerencial para RSU, acá se articula la función gestión a través de los diferentes procesos implicados en RSU (ver Tabla 6). Se plantea cómo funciones universitarias deben estar al servicio del entono social, así como un majeño responsable socialmente en universidades a lo interno para cumplir ese rol no solo con el contexto sino con los propios integrantes de una comunidad universitaria, llámense docentes, administrativos, obreros y estudiantes. En esta parte de lo encontrado a partir de voces de informantes clave es innovadora, pues se reivindica el rol amplio de RSU, al no ser solo un proceso de acción a lo externo, sino con impacto de forma integral a la organización.

Tabla 6

Quinta categoría emergente

\begin{tabular}{|c|c|c|}
\hline Categorías & Subcategorías & Hallazgos \\
\hline \multirow{3}{*}{$\begin{array}{l}\text { Praxis gerencial para } \\
\text { RSU (PGRSU) }\end{array}$} & $\begin{array}{l}\text { Procesos gerenciales universitarios } \\
\text { (PGU) }\end{array}$ & $\begin{array}{l}\text { - Gerentes deben identificar necesidades del } \\
\text { entorno } \\
\text { - Preparación y actualización de los gerentes }\end{array}$ \\
\hline & & 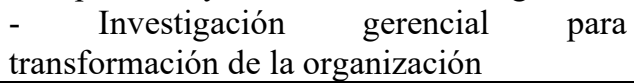 \\
\hline & $\begin{array}{l}\text { Proyección institucional de } \mathrm{RSU} \\
\text { (PIRSU) }\end{array}$ & $\begin{array}{l}\text { - Lo no se divulgado no se conoce } \\
\text { - Opiniones de comunidades sobre la } \\
\text { universidad }\end{array}$ \\
\hline
\end{tabular}

Nota. Representación de proceso de categorización emergente, elaboración propia (2021).

Ahora bien, los procesos gerenciales en RSU deben venir declarados en la filosofía de gestión de cada universidad, así como en los planes estratégicos, para definir políticas que se van a realizar en esta materia. En este sentido, lo presentado se vincula al planteamiento de Wigmore (2016) quien observó en su estudio de un interés en ser socialmente responsable por parte de universidades. Dicha afirmación se presenta en la Universidad Yacambú, pues los procesos gerenciales de alto nivel están orientados hacia una formación en valores tanto institucionales como globales, anclados a políticas específicas. Además, como parte de una praxis gerencial, la investigación debe servir para transformación de la organización, a los fines que permita ese intercambio permanente de información, para así divulgar los alcances en materia de RSU manteniendo una opinión favorable de comunidades hacia la universidad.

Como aporte teórico a las ideas desarrolladas sobre los procesos gerenciales, Goyo et. al discuten como universidades son “... espacios gerenciales multidinámicos $\mathrm{y}$ transcomplejos por los procesos que allí se desarrollan" (2012, p.115), donde cada uno es interdependiente del otro, al funcionar como un sistema organizado, con fines establecidos, pero con un común en su rol transformador de sociedades, donde tecnologías como medio para la educación han sido un pilar 
fundamental para responder a esta situación de pandemia por COVID-19.

Como segunda subcategoría, praxis gerencial para RSU se refiere a una proyección institucional que debe existir cuando se trabaja con responsabilidad social universitaria, es decir, promoción de actividades desarrolladas en ese marco procedimental. En este sentido, el planteamiento anterior se relaciona con Rivero et. al, quien indica "La gerencia universitaria debe estar acorde con lo moderno, emprendedora, eficaz, innovadora, revolucionaria, con muchos más apelativos que apuntan a exhibir su eficiencia $y$ potencia" (2012, p.22), esto significa a hacer el uso de los diferentes recursos tecnológicos, sobre todo las redes sociales para mantener su proyección.

Como última categoría emergente del intercambio dialógico con informantes clave, surgió "Educación mediada por las tecnologías", resume los aspectos propios de una parte del fenómeno de estudio como es esa educación que ha dado respuestas ante esta situación sanitaria vivida a lo largo de los dos últimos años, al respecto se tiene:

La pandemia de enfermedad por coronavirus (COVID-19) ha provocado una crisis sin precedentes en todos los ámbitos. En la esfera de la educación, esta emergencia ha dado lugar al cierre masivo de las actividades presenciales de instituciones educativas en más de 190 países con el fin de evitar la propagación del virus y mitigar su impacto. Según datos de la Organización de las Naciones Unidas para la Educación, la Ciencia y la Cultura (Unesco), a mediados de mayo de 2020 más de 1.200 millones de estudiantes de todos los niveles de enseñanza, en todo el mundo, habían dejado de tener clases presenciales en la escuela (CEPALUnesco, 2020, p.1)

Como se evidencia en lo señalado por el informe de CEPAL-Unesco en agosto de dos mil veinte, los procesos educativos han tenido ajustes vinculados a medidas tomadas por los países como procedimiento de resguardo y mitigación de los contagios del virus SarsCov-2. En este sentido, en Tabla 7 se presentan subcategorías y hallazgos emergidos de las voces de informantes clave en torno al proceso de educación mediada por tecnología en la Universidad Yacambú.

\section{Tabla 7}

\begin{tabular}{|c|c|c|}
\hline Categorías & Subcategorías & Hallazgos \\
\hline \multirow[t]{2}{*}{$\begin{array}{l}\text { Educación } \\
\text { mediada } \\
\text { por } \\
\text { tecnologías } \\
\text { (EMT) }\end{array}$} & $\begin{array}{l}\text { Vinculación de } \\
\text { TIC en } \\
\text { funciones } \\
\text { universitarias } \\
\text { (VTFU) }\end{array}$ & \begin{tabular}{lr}
\multicolumn{1}{c}{ Redes } & de \\
investigación & \\
- Apoyo a otras \\
instituciones \\
el con \\
plataforma \\
EDUNY \\
- Creación \\
recursos \\
multimedia \\
- Plataformas de \\
videoconferencias \\
- Grupos \\
WhatsApp de \\
atención \\
comunitaria
\end{tabular} \\
\hline & $\begin{array}{lr}\text { Calidad de } \\
\text { educación a } \\
\text { distancia en la } \\
\text { UNY } \\
\text { (CEDUNY) }\end{array}$ & $\begin{array}{lr}\text { - Hay un camino } \\
\text { trazado } \\
\text { educación } \\
\text { distancia } \\
\text { - Ejemplo de } \\
\text { gestión educativa } \\
\text { a distancia en el } \\
\text { país }\end{array}$ \\
\hline
\end{tabular}

Nota. Representación de proceso de categorización emergente, elaboración propia (2021).

La subcategoría vinculación de TIC en funciones universitarias se articula de forma directa con dimensiones de RSU pues abarca procesos inmersos en docencia, investigación y extensión. Específicamente producto de una situación de pandemia, la universidad ha prestado apoyo, a través de formación a otras instituciones educativas de diferentes niveles a través de su plataforma de gestión educativa a distancia denominada EDUNY. Por su parte para existe vinculación comunitaria a través los grupos de comunicación con el uso de aplicación móvil WhatsApp. También destaca implementación de plataformas de videoconferencias, inicialmente Bigbluebutton, posteriormente con Zoom. Al respecto, de acuerdo con el informe " $L a$ educación en tiempos de la pandemia de COVID-19" 
Entre las modalidades de aprendizaje a distancia en línea destaca el uso de plataformas virtuales de aprendizaje asincrónico, utilizadas en 18 países, en tanto que solo 4 países ofrecen clases en vivo... A su vez, entre las formas de aprendizaje a la distancia fuera de línea, 23 países realizan transmisiones de programas educativos por medios de comunicación tradicionales como la radio o la televisión (CEPAL-Unesco, 2020, p.3)

De acuerdo con García "la pandemia COVID-19 ha venido generando cambios $\mathrm{y}$ disrupciones en amplios sectores de la actividad humana... La modalidad de educación a distancia, fundamentalmente en soporte digital, vino a ofrecer soluciones de emergencia a dicha crisis" $(2021$, p. 9). Este hecho es un punto a favor en la Universidad Yacambú, reflejado como segunda subcategoría denominada "Calidad de la educación a distancia en la UNY". Además, García indica: "Sin duda, las universidades, ... lo tuvieron y lo tienen más fácil. En primer lugar, porque muchas de ellas ya contaban con plataformas digitales que venían utilizando en algunas fases del desarrollo de su docencia, generalmente presencial" (2001, p.11), lo que sustenta en los hallazgos obtenidos, al tener como institución un largo camino trazado en educación a distancia, por ello se ha convertido en un ejemplo de gestión educativa a distancia en el país.

Luego de realizado el proceso de develar, comprender e interpretar los significados sobre responsabilidad social universitaria (RSU) desde una educación mediada por tecnología producto de la pandemia COVID-19, partiendo de voces de informantes clave con apoyo de literatura vinculada con el objeto de estudio, emergieron seis categorías y trece subcategorías, todas orientadas hacia una comprensión del fenómeno, a fin de conocer su constitución para lograr funcionar como procesos interdisciplinarios a estudiarse desde diferentes ángulos y áreas de aplicación.

\section{Conclusiones}

Las dimensiones de responsabilidad social universitaria están definidas por diversos autores, agrupadas en docencia, investigación, extensión y gestión, pero en el discurrir hermenéutico se encontró con constructos que no encajaban fácilmente en dichas dimensiones. Por ejemplo, el estudiante dentro del contexto comunitario, debido a su participación en todas las facetas de una dinámica universitaria, además son los protagonistas del acto educativo tanto en el nivel de pregrado como en el de postgrado.

El papel de la investigación en el desarrollo del proceso de RSU es indiscutible, pues los problemas sociales declarados por la Universidad Yacambú han sido producto de una aplicación de diferentes instrumentos diagnósticos; donde lo tecnológico ha sido un pilar fundamental al proporcionar tanto formas de rápido acceso como procesamiento de información. Existen competencias tecnológicas inmersas en el modelo educativo vigente que demuestran cómo el docente y el estudiante son capaces de aplicarlas para fortalecer sus habilidades instrumentales.

La docencia en tiempos de pandemia por COVID-19 a través del uso de TIC no ha representado un reto para la Universidad Yacambú, pues en su transitar tiene una cantidad considerable de tiempo desarrollando como modalidad a distancia, donde los entornos virtuales de aprendizaje, representados por una plataforma "EDUNY", ha sido el espacio de encuentro asíncrono y síncrono entre docentes-estudiantes para formar profesionales, líderes, emprendedores con sentido humano, sensibilidad ambiental, así como competentes para desenvolverse en escenarios globales.

La situación sanitaria mundial vivida desde el año 2020 hasta el día de hoy, ha llevado al desarrollo tanto de los procesos de extensión como de atención social por parte de la institución se apoyen en TIC disponibles en los escenarios comunitarios. De forma tal, que docentes y estudiantes puedan tengan ese contacto necesario en su proceso de formación integral como profesionales en ejercicio, futuros profesionales en una carrera determinada. Asimismo, una experiencia 
relevante; el servicio comunitario, es un proceso desarrollado de forma continua a pesar del COVID-19, siendo gestionado desde un enfoque más local a través de mediación por TIC.

La responsabilidad social universitaria desde un enfoque educativo mediado por tecnologías producto en torno al contexto de pandemia por COVID-19 en la Universidad Yacambú ha sido un proceso de constante adecuación a necesidades, recursos disponibles, manteniendo siempre activas sus funciones de docencia, investigación, extensión y gestión. Lo cual se traduce en una evolución hacia una nueva perspectiva educativa en contextos complejos, de alta incertidumbre, donde prevalece una mejora constante para lograr ser una institución competitiva, de calidad reconocida tanto a nivel nacional como internacional.

Finalmente, producto de los hallazgos se recomienda realizar investigaciones que compare el proceso de responsabilidad social universitaria trabajado en la modalidad tradicional en relación con educación mediada por tecnologías para así evocar puntos de similitud o diferencia. Además, valorar experiencias tanto de docentes como estudiantes en su gestión académica desde una perspectiva que incluya a la docencia, investigación y extensión.

\section{Referencias}

Baca, H. (2015). La responsabilidad social universitaria: propuesta conceptual y medición en el ámbito de una Universidad Privada de Lima - Perú. [Tesis Doctoral, Universidad de Sevilla]. https://idus.us.es/handle/11441/38435

CEPAL-Unesco. (2020). La educación en tiempos de la pandemia de COVID-19. Digital Repository Economic Commission for Latin America and the Caribbean

https://repositorio.cepal.org/bitstream/handle/11362/ 45904/1/S2000510_es.pdf

Constitución de la República Bolivariana de Venezuela. (1999). Gaceta Oficial de la República Bolivariana de Venezuela, $N^{\circ} 36.860 \quad$ (Extraordinaria), Diciembre 30, 1999.

García, L. (2021). COVID-19 y educación a distancia digital: preconfinamiento, confinamiento y posconfinamiento. Revista Iberoamericana de
Educación a Distancia, 24 (1), 9-25. https://www.redalyc.org/jatsRepo/3314/3314644600 01/331464460001.pdf

Goyo, A., Figueredo, C., Méndez, E., Chirinos, E. y Rivero, E. (2012). La gerencia de la educación universitaria, en la perspectiva de la transcomplejidad. Revista Científica Ciencias Humanas, 8 (23), 109-131. http://www.redalyc.org/pdf/709/70925416004

Hernández, N. (2014). Teoría de la gestión del conocimiento. Gestiopolis. https://www.gestiopolis.com/teoria-dela-gestion-del-conocimiento/

Martínez, M. (1991). La investigación cualitativa etnográfica en educación: Manual teórico práctico. Venezuela.

Martínez, M. (2006). Ciencia y arte en la metodología cualitativa. Trillas.

Muñoz, C. (2012). Responsabilidad Social Universitaria: Aportes al enriquecimiento del concepto desde la dimensión de las prácticas. Instituto Universitario de Desarrollo y Cooperación - Universidad Complutense de Madrid. https://www.ucm.es/data/cont/docs/599-2013-1116-Doc_23.pdf

Núñez, C., Gaviria-Serrano, J., Tobón, S., Guzmán-Calderón, C. \& Herrera, S. (2019). La práctica docente mediada por TIC: una construcción de significados. Espacios, 40(5), 1-15. http://w.revistaespacios.com/a19v40n05/a19v40n05 p04.pdf

Okuda, M. \& Gómez-Restrepo, C. (2005). Métodos en investigación cualitativa: triangulación. Revista Colombiana de Psiquiatría, 34(1), 118-124. $\mathrm{http}: / /$ www.scielo.org.co/scielo.php?script=sci_artte xt\&pid=S0034-74502005000100008

Orellana, G. (2015). La praxis pedagógica del docente de biología del Instituto Pedagógico de Barquisimeto (IPB). $\quad$ Educere, $19(62), \quad 91-99$. https://www.redalyc.org/pdf/356/35641005008.pdf

Pérez, G. (1994). Investigación cualitativa: retos $e$ interrogantes. Muralla.

Rivero, E. \& Goyo, A. (2012). La gerencia universitaria venezolana ante los nuevos retos de la sociedad del siglo XXI. Gestión y Gerencia, 6 (2), 4-25. http://www.ucla.edu.ve/DAC/investigacion/gyg/Gy G\%202012/Agosto\%202012/1$\%$ 20EduardaRiveroyOtros.pdf

Vallaeys, V. (2007). Responsabilidad Social Universitaria. Propuesta para una definición madura y eficiente. Biblioteca Virtual en Responsabilidad Social y temas relacionados.

http://www.bibliotecavirtualrs.com/wpcontent/uploads/2011/12/Responsabilidad_Social_U niversitaria_Francois_Vallaeys.pdf

Vallaeys, F. (2014). La responsabilidad social universitaria: 
un nuevo modelo universitario contra la mercantilización. Universia, 12(5), 105-117. http://www.scielo.org.mx/scielo.php?script=sci_artt ext\&pid=S2007-28722014000100006

Wigmore, A. (2016). La gestión de la Responsabilidad Social Universitaria (RSU). [Tesis Doctoral, Universidad de Córdova].

http://helvia.uco.es/bitstream/handle/10396/13365/2

016000001394.pdf? sequence $=1$ \&isAllowed $=\mathrm{y}$ 